\title{
Side differences of the lateral abdominal wall in supine rest position in mild adolescent idiopathic thoracolumbar scoliosis
}

\author{
Pawel Linek, Tomasz Wolny, Edward Saulicz, Andrzej Mysliwiec \\ Department of Kinesitherapy and Special Methods in Physiotherapy, The Jerzy Kukuczka Academy of Physical Education in Katowice, Katowice, Poland
}

Received: May 2016 Accepted: August 2016

\begin{abstract}
Objectives: This study aims to evaluate the thickness of the abdominal muscles on both sides in patients with mild adolescent idiopathic scoliosis (AIS) and to assess the absolute and relative thickness of oblique external (OE), oblique internal (OI), and transversus abdominis (TrA).

Patients and methods: A total of 84 participants underwent ultrasonographic examination of the abdominal muscles in the supine resting position. All participants were divided into two groups including AIS group $(n=42)$ and control group $(n=42)$. The absolute and relative thicknesses of OE, OI, and TrA were recorded.

Results: In the AIS group, the TrA on the left side was significantly thicker by $0.30 \mathrm{~mm}$ (95\% CI 0.01-0.7) than the right side. For relative values, the percentage contribution to the structure of the lateral abdominal wall of the OE on the right and the TrA on the left was significantly higher by $3.2 \%$ (95\% CI 0.9-5.5) and 3.1\% (95\% CI 1.1-5.0), respectively, in the AIS group.

Conclusion: Our study results show that, in the supine resting position, the muscles of the lateral abdominal wall are thinner in AIS patients. In addition, side-to-side differences in the percentage contribution of the OE and TrA to the structure of the lateral abdominal wall are seen in this patient population, although these differences are independent of the direction of the scoliosis.
\end{abstract}

Keywords: Abdominal muscles; scoliosis; ultrasonography.

Thoracolumbar scoliosis is a common type of adolescent idiopathic scoliosis (AIS), which is a threedimensional deformity of the spine. Some authors suggest that deformity of the spine may be a result of the weakening or inappropriate functioning of the abdominal muscles. ${ }^{[1,2]}$ Biomechanical studies have shown that the abdominal muscles play a significant role in the stabilization of the spine. ${ }^{[3]}$ The transversus abdominis ( $\operatorname{Tr} \mathrm{A})$, the deepest abdominal muscle, is responsible for maintaining the neutral curvature and stiffness of the lumbar spine, and the more superficial oblique external (OE) and internal muscles function depending on the external forces created and generates a torque to preserve the stability of the trunk. ${ }^{[4]}$

Ultrasonography (USG) has often been used for the quantitative assessment of the lateral abdominal wall in a healthy population and in individuals with low back pain..$^{[5-8]}$ It has been demonstrated that the muscle thickness quantified by USG is associated with the level of activity as assessed by electromyography and is similar to the muscle thickness estimated by magnetic resonance imaging. ${ }^{[9,10]}$ Therefore, the size of the muscle may provide an indirect measurement of force-generating capacity, as demonstrated for various muscles. ${ }^{[1]}$

To the best of our knowledge, there is a lack of studies about the characteristic of the lateral abdominal muscles in AIS patients. The only available studies have demonstrated that the thickness of all lateral abdominal muscles in the supine rest position is different in AIS patients. ${ }^{[2,12]}$ In the reports published, however, the authors found no possible side-to-side differences between the body sides. However, the appearance of any side-to-side differences in the thickness of the individual abdominal muscles during the stabilization of the trunk can induce a muscle

Corresponding author: Pawel Linek, MD. Department of Kinesitherapy and Special Methods in Physiotherapy, The Jerzy Kukuczka Academy of Physical Education in 40-065, Mikolowska 72B, Katowice, Poland. e-mail: linek.fizjoterapia@vp.pl 
imbalance that, in turn, causes micro-injuries in the facet joints or intervertebral discs. ${ }^{[13]}$ This has been investigated as the reason for the development of AIS

The USG studies confirm the asymmetry and morphological changes in the paraspinal muscles in AIS patients. ${ }^{[14]}$ Therefore, in the present study, we aimed to evaluate the thickness of the abdominal muscles on both sides in patients with mild adolescent idiopathic thoracolumbar scoliosis and to answer the following questions: Are there any differences in the absolute thickness of specific abdominal muscles? Are there any differences in the percentage of the contribution of specific muscles to the building of the lateral wall of the abdomen? Are any side-to-side differences associated with the direction of scoliosis?

\section{PATIENTS AND METHODS}

\section{Subjects}

A total of 84 participants was divided into two groups: 42 adolescents without scoliosis (control group) and 42 patients with AIS (AIS group). Participants in the control group were recruited randomly from public schools, whereas the patients with AIS were recruited from a corrective exercise center. The control group included individuals without any signs of scoliosis upon screening and no trunk rotation in the Adam's forward bend test using a scoliometer, which is used in clinical studies of children with scoliosis. ${ }^{[15]}$ The AIS group included individuals in whom an orthopedist diagnosed single-curve idiopathic scoliosis, in which the apex was at the thoracic spine, level 12 (Th12), L1, or at the intervertebral Th12/L1 disc level (confirmed by $\mathrm{X}$-ray), i.e., thoracolumbar scoliosis according to the Ponseti's classification. ${ }^{[16]}$ According to the Guidelines of the International Scientific Society on Scoliosis Orthopedic and Rehabilitation Treatment, a $5^{\circ}$ error in the measurements of Cobb angle should be taken into account; therefore, those with a Cobb angle of $\geq 5^{\circ}$ were included in this study ${ }^{[17]}$ All subjects (in both groups) were free of any documented spinal surgery or other surgical treatment, and any cardiovascular or neurological disease. A written informed consent was obtained from each participant. The study protocol was also approved by the local Ethics Committee. The study was conducted in accordance with the principles of the Declaration of Helsinki.

\section{USG examination}

A real-time USG B scanner (Mindray DP-6600 Digital Ultrasonic Diagnostic Imaging System) (Medical Corp., Redmond, USA) with a $60 \mathrm{~mm}$ wide 75L38EA linear array transducer (5.0/7.5/10 $\mathrm{MHz}$ ) was used to obtain images of the muscles. The penetration depth was $5.39 \mathrm{~cm}$ at a sampling frequency of $7.5 \mathrm{MHz}$. The transducer was always placed on the anterolateral wall of the abdomen, between the iliac crest and the inferior boundary of the $12^{\text {th }}$ rib, perpendicular to the longitudinal axis of the body. The transducer was also always placed at the same altitude and distance from the musculofascial junction of the $\operatorname{Tr} A$ during examination on the right and left body side of a subject.

Measurements of the muscle thickness were collected in the supine position, where the knees were extended, the upper limbs were placed along the sides of the trunk, and the head was positioned on a pillow. The thickness was measured in millimeters $(\mathrm{mm})$ at the end of normal expiration. All images were captured and measured offline. In previous studies, the reliability of the USG of the lateral abdominal wall in adolescents was evaluated, and analysis revealed that the use of the mean value of three measurements was characterized by high reliability. ${ }^{[6-8,18]}$

\section{Statistical analysis}

The t-test for independent measurements was used to compare basic parameters including age, height, and body weight in the population examined. The mean values from three trials for a given person on a given side were used for further analysis. Descriptive data were expressed in mean and standard deviation (SD). 95\% confidence interval (CI) for absolute and relative muscle sizes was calculated. Relative thickness of each muscle was expressed in percentage of the total muscle thickness.

The homogeneity of variance was checked and subsequently confirmed using the Levene's test. Differences between the groups (control vs AIS or scoliosis right-handed vs scoliosis left-handed) and body side (right vs left) in each case were examined using two-way analysis of variance (ANOVA) using two between-group factors. In the presence of a significant effect, a post-hoc Tukey analysis was performed. For each ANOVA component (main effect or interaction), eta-squared effect size and test power were computed. Eta-squared is a measure of effect size calculated according to the guidelines of Cohen $(0.01=$ small effect, $0.06=$ moderate effect, $0.14=$ large effect). A $p$ value of $<0.05$ was considered statistically significant. All data were analyzed using the Statistica software version 12PL (StatSoft, Inc.; Tulsa, OK, USA). 


\section{RESULTS}

\section{Subjects}

In the examined population, there were no significant differences between the two groups for any of basic variables (Table 1). Single-curve thoracolumbar scolioses were, however, detected in the AIS group. In $64.3 \%$ of the patients, left scoliosis was found with a mean Cobb angle of $10.7 \pm 3.3^{\circ}$. The other $35.7 \%$ of the patients had right scoliosis with a mean value of $10 \pm 3.4^{\circ}$. No significant differences in the Cobb angles were found between right and left scoliosis ( $p>0.05$ ).

\section{Absolute muscle thickness}

There was a significant difference in the OE, OI, and TrA thickness between the groups (Table 2). For the OE thickness, the post-hoc analyses revealed that the OE was thicker by $0.98 \mathrm{~mm}$ in the control group (95\% CI 0.6-1.3) than the AIS group. For the OI thickness, the post-hoc analysis demonstrated that the OI was thicker by $1.7 \mathrm{~mm}$ in the control group (95\% CI 1.2-2.1) than the AIS group. Additionally, the TrA in the control group was thicker by 0.81 (95\% CI 0.5-1.1) than the AIS group. However, there was no significant difference between the body sides (Figure 1a).
The main effect of the scoliosis direction and the interaction (between the direction of the scoliosis and body side) did not significantly affect the thickness of any abdominal muscles examined (Table 3). Only the main effect of the body side was found to be significant for the OE and TrA. The OE on the right side was thicker by $0.4 \mathrm{~mm}$ (95\% CI $0.03-0.8)$ and the $\operatorname{TrA}$ on the right side was thinner by $0.3 \mathrm{~mm}$ (95\% CI 0.01-0.7). There was no significant difference between the scoliosis directions (Figure 2).

\section{Relative muscle thickness}

Significant interactions between the body side and the group were observed in terms of the percentage contribution of the OE and TrA to the building of the lateral wall of the abdomen (Table 2). As demonstrated by the post-hoc analysis, the percentage contribution of the OE on the right was higher by $3.2 \%$ (95\% CI 0.9-5.5) than the left, and the percentage contribution of the TrA on the left was higher by $3.1 \%$ (95\% CI 1.1-5.0) than the right. There were significant differences only in the AIS group (Figure 1b).

Table 3 shows that only the main effect of the body side proved significant for the $\mathrm{OE}$ and $\operatorname{TrA}$, and the OE on the right side and the TrA on the left side had a higher percentage contribution to the building of

Table 1. Demographic characteristics of adolescent idiopathic scoliosis patients and control subjects

\begin{tabular}{|c|c|c|c|c|c|c|c|c|}
\hline \multirow[b]{2}{*}{ Variables } & \multicolumn{3}{|c|}{ Control groups $(n=42)$} & \multicolumn{3}{|c|}{ AIS groups $(n=42)$} & \multicolumn{2}{|c|}{ Difference between groups } \\
\hline & $\mathrm{n}$ & $\%$ & Mean \pm SD & $\mathrm{n}$ & $\%$ & Mean \pm SD & t-test & $p$ level \\
\hline Age (year) & & & $12.4 \pm 1.5$ & & & $12.5 \pm 1.8$ & -0.2 & 0.8 \\
\hline Weight (kg) & & & $48.1 \pm 12.7$ & & & $46.9 \pm 11.8$ & 0.4 & 0.7 \\
\hline Height $(\mathrm{cm})$ & & & $158.4 \pm 11.1$ & & & $157.5 \pm 12.3$ & 0.3 & 0.7 \\
\hline Number of boys (\%) & 27 & 64.3 & & 27 & 64.3 & & & \\
\hline Number of girls (\%) & 15 & 35.7 & & 15 & 35.7 & & - & $1.0 \dagger$ \\
\hline
\end{tabular}

SD: Standard deviation; $\uparrow$ Differences between the groups was evaluated using the Fisher's exact test.

Table 2. Comparison of muscle thickness between adolescent idiopathic scoliosis and control groups

\begin{tabular}{|c|c|c|c|c|c|c|}
\hline & \multicolumn{3}{|c|}{$\mathrm{p}$ value from ANOVA } & \multicolumn{3}{|c|}{ Eta-squared/power } \\
\hline & \multicolumn{2}{|c|}{ Main effect } & \multirow[b]{2}{*}{ Interaction } & \multicolumn{2}{|c|}{ Main effect } & \multirow[b]{2}{*}{ Interaction } \\
\hline & Group & Body site & & Group & Body site & \\
\hline \multicolumn{7}{|l|}{$\mathrm{OE}$} \\
\hline Rest (mm) & $<0.001^{\star}$ & 0.09 & 0.38 & $0.19 / 0.99$ & $0.02 / 0.39$ & $<0.01 / 0.14$ \\
\hline Relative (\%) & 0.78 & $0.03^{*}$ & $<0.01^{\star}$ & $<0.01 / 0.06$ & $0.03 / 0.61$ & $0.05 / 0.84$ \\
\hline \multicolumn{7}{|l|}{ OI } \\
\hline Rest (mm) & $<0.001^{*}$ & 0.20 & 0.33 & $0.22 / 0.99$ & $0.01 / 0.24$ & $<0.01 / 0.16$ \\
\hline Relative (\%) & 0.87 & 0.46 & 0.34 & $<0.01 / 0.06$ & $<0.01 / 0.11$ & $<0.01 / 0.16$ \\
\hline \multicolumn{7}{|l|}{$\operatorname{TrA}$} \\
\hline Rest (mm) & $<0.001^{\star}$ & 0.45 & 0.15 & $0.19 / 0.99$ & $<0.01 / 0.12$ & $0.01 / 0.31$ \\
\hline Relative (\%) & 0.60 & $<0.001^{\star}$ & $0.03^{*}$ & $<0.01 / 0.08$ & $0.07 / 0.94$ & $0.02 / 0.59$ \\
\hline
\end{tabular}


(a)

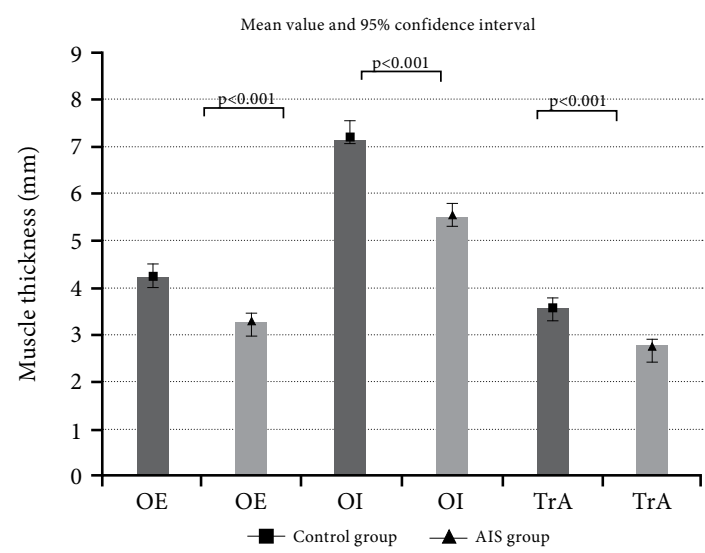

(b)

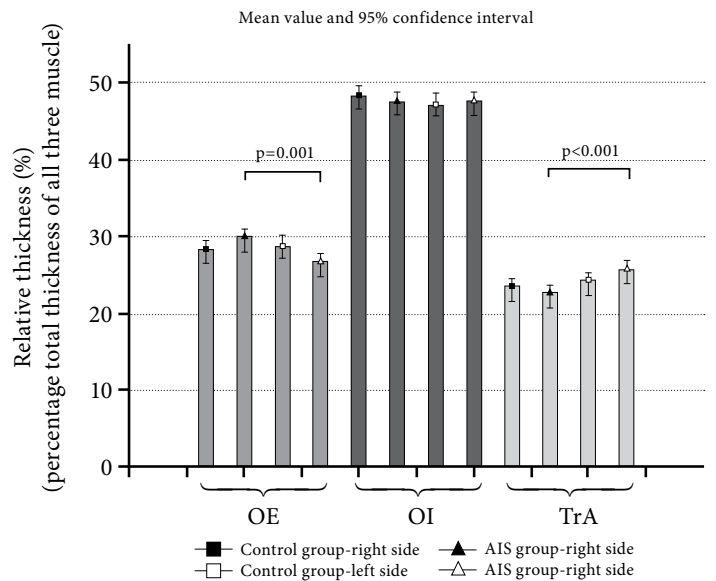

Figure 1. Absolute and relative muscle thickness in control and adolescent idiopathic scoliosis groups. OE: Oblique external; OI: Oblique internal; TrA: Transversus abdominis.

Table 3. Comparison of muscle thickness between right-handed and left-handed scoliosis

\begin{tabular}{|c|c|c|c|c|c|c|}
\hline & \multicolumn{3}{|c|}{$\mathrm{P}$ value from ANOVA } & \multicolumn{3}{|c|}{ Eta-squared/power } \\
\hline & \multicolumn{2}{|c|}{ Main effect } & \multirow[b]{2}{*}{ Interaction } & \multicolumn{2}{|c|}{ Main effect } & \multirow[b]{2}{*}{ Interaction } \\
\hline & Group & Body site & & Group & Body site & \\
\hline \multicolumn{7}{|l|}{$\mathrm{OE}$} \\
\hline Rest (mm) & 0.72 & $0.02^{\star}$ & 0.47 & $<0.01 / 0.06$ & $0.06 / 0.62$ & $<0.001 / 0.11$ \\
\hline Relative (\%) & 0.71 & $<0.001^{\star}$ & 0.25 & $<0.01 / 0.06$ & $0.18 / 0.99$ & $0.01 / 0.21$ \\
\hline \multicolumn{7}{|l|}{ OI } \\
\hline Rest (mm) & 0.97 & 0.79 & 0.90 & $<0.01 / 0.05$ & $<0.01 / 0.06$ & $<0.01 / 0.05$ \\
\hline Relative (\%) & 0.95 & 0.72 & 0.49 & $<0.01 / 0.05$ & $<0.01 / 0.06$ & $<0.01 / 0.11$ \\
\hline \multicolumn{7}{|l|}{$\operatorname{TrA}$} \\
\hline Rest (mm) & 0.66 & $0.05^{\star}$ & 0.92 & $<0.01 / 0.07$ & $0.04 / 0.49$ & $<0.01 / 0.05$ \\
\hline Relative (\%) & 0.66 & $<0.001^{\star}$ & 0.73 & $<0.01 / 0.07$ & $0.15 / 0.95$ & $<0.01 / 0.06$ \\
\hline
\end{tabular}

OE: Obliquus externus abdominis; ${ }^{*}$ Statistically significant difference; OI: Obliquus internus abdominis; TrA: Transversus abdominis. Relative thickness expressed in percentage of total thickness of all three muscles. $P$ value, Eta-squared and power from analysis of variance.

the lateral wall of the abdomen by $3.2 \%$ and by $3.1 \%$, respectively. The results were already confirmed in earlier analyses (right side; Figure 1).

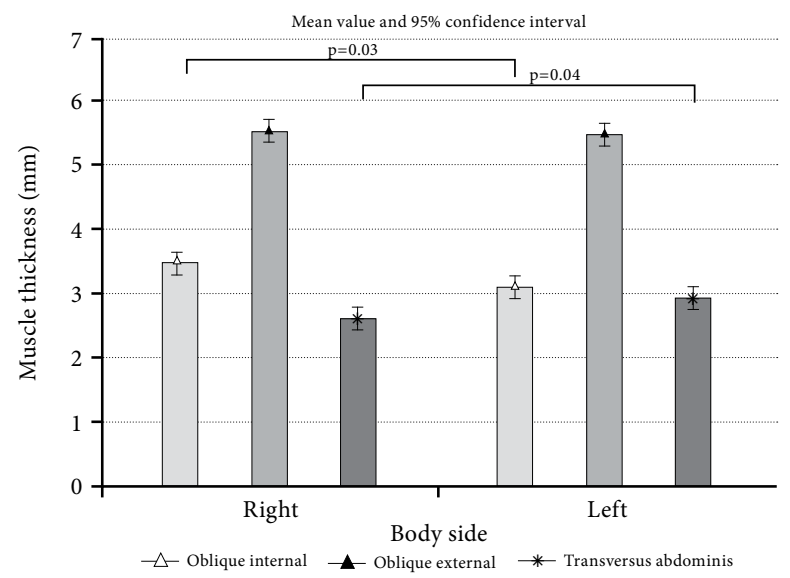

Figure 2. Absolute muscle thickness on both body sides in adolescent idiopathic scoliosis group.

\section{DISCUSSION}

The primary goal of this study was to make a side-to-side comparison of the thickness (actual and relative) of the lateral abdominal muscles in adolescents with a mild thoracolumbar scoliosis compared to control group. Our study results indicated that, among all the lateral abdominal muscles, the muscles with a significant side-to-side difference were the $\mathrm{OE}$ and TrA in the AIS group. In addition, the analysis of the relative thickness showed that, in the AIS group, the percentage contribution to the building of the lateral abdominal wall of the $\mathrm{OE}$ on the right and TrA on the left was more than $3 \%$, indicating a higher ratio than the contribution of the corresponding muscles on the other side. However, such differences were not observed in the control group. In addition, we found that the OE, OI, and TrA on both sides are significantly thinner in the AIS group.

To the best of our knowledge, it is difficult to explain why the lateral abdominal muscles are thinner 
with varying percentage contribution and higher asymmetry of the OE and TrA muscle thickness in AIS patients. This is due to the lack of studies on the role and function of the abdominal muscles in AIS patients. The available data have shown that the body weight in adolescents has the highest effect on muscle thickness, and is the most consistent unique predictor of $\mathrm{OE}$, OI, and TrA thickness variance, while the second best predictor is sex..$^{[6]}$ In this study, body weight was taken into account and its distribution was similar in both groups studied. Sex was also considered (the ratio of the number of boys to girls was identical in both groups). It is also well-known that practicing sport and physical activity significantly affect the thickness of the lateral abdominal muscles. ${ }^{[19,20]}$ However, the level of physical activity of the individuals was unable to be evaluated. The AIS group was composed of individuals who regularly participated in obligatory corrective exercises; despite this, their abdominal muscles were significantly thinner than those of the control group.

Considering that the size of the muscle may provide an indirect measurement of force-generating capacity, ${ }^{[1]}$ we can hypothesize that the muscles of the lateral abdominal wall cannot generate sufficient force to stabilize the pelvis and spine during various daily activities in AIS patients, as $\operatorname{TrA}$ generates $40 \%$ of the force necessary for the tension of the thoracolumbar fascia and along with the OI and significantly increases the stabilization of the sacroiliac joints. ${ }^{[21]}$ This issue has already been addressed in the studies in which similar differences in the thickness of the abdominal muscles were noticed in AIS patients. However, unlike in this report, different types of scoliosis were analyzed together. ${ }^{[2]}$

Regarding the characteristics of scoliosis, side-toside differences may prove to be more important in terms of the absolute and relative abdominal muscle thicknesses. The normative data on a healthy adolescent population indicated that there are no significant sideto-side differences in the lateral abdominal muscle thicknesses. ${ }^{[6]}$ Thus, the asymmetry in the OE and TrA thickness in the AIS group has shown changes in characteristic of the lateral abdominal wall in the AIS population. In addition, if percentage relative values of the thickness of lateral abdominal muscles are compared, next significant differences are observed in the AIS group, in which the percentage contribution of the OE on the right and $\operatorname{TrA}$ on the left side is higher. Such differences were not observed in the control group or in the studies performed in the healthy population.

${ }^{[6]}$ More interestingly, the differences for both muscles are similar. It seems that in AIS patients, a greater percentage role of the TrA on the left is compensated by the OE on the right (or vice versa). However, no significant differences were observed in the AIS group between the directions of scoliosis, which does not support a possible theory of a specific (associated with the direction of the scoliosis) type of abdominal muscle asymmetry, as in case of the multifidus muscle. ${ }^{[14]}$

Due to a significant lack of studies of the structure of the abdominal muscles in AIS patients and due to the results of the current studies, further studies in this area are required. An analysis of more advanced scoliosis with different locations would probably reveal certain unequivocal asymmetry patterns which can contribute to a better understanding of this disorder. It would be also worthwhile to conduct this type of analysis at higher body positions, where the reflex activity of the abdominal muscles studied is higher. Nonetheless, these studies are associated with certain limitations including lack of $\mathrm{X}$-rays in the control group, the lack of information concerning physical activity of the participants, and mild scoliosis degree in the AIS group. In certain situations (in which the Cobb angle was below a 10 degree) scoliotic postures rather than scoliosis were examined. Additionally, it is likely that, in AIS patients with a higher Cobb degree, the lateral abdominal muscles can be more affected. However, our study allows us to suppose that the changes in the lateral abdominal muscles in patients with spinal deviation are prior to structural changes, which are typical for more advanced scoliosis.

In conclusion, in the supine resting position, the muscles of the lateral abdominal wall are thinner in AIS patients. In addition, side-to-side differences in the percentage contribution of the $\mathrm{OE}$ and $\mathrm{TrA}$ to the structure of the lateral abdominal wall are seen in this patient population. However, these differences are independent of the direction of the scoliosis.

\section{Declaration of conflicting interests}

The authors declared no conflicts of interest with respect to the authorship and/or publication of this article.

\section{Funding}

The authors received no financial support for the research and/or authorship of this article.

\section{REFERENCES}

1. Lam KS, Mehdian H. The importance of an intact abdominal musculature mechanism in maintaining spinal sagittal balance. Case illustration in prune-belly syndrome. Spine (Phila Pa 1976) 1999;24:719-22. 
2. Linek P, Saulicz E, Kuszewski M, Wolny T. Ultrasound Assessment of the Abdominal Muscles at Rest and During the ASLR Test Among Adolescents with Scoliosis. Clin Spine Surg 2016 Jun 3. [Epub ahead of print]

3. Sharpe SR, Holt KG, Saltzman E, Wagenaar RC. Effects of a hip belt on transverse plane trunk coordination and stability during load carriage. J Biomech 2008;41:968-76.

4. Bergmark A. Stability of the lumbar spine. A study in mechanical engineering. Acta Orthop Scand Suppl 1989;230:1-54.

5. Pulkovski N, Mannion AF, Caporaso F, Toma V, Gubler $\mathrm{D}$, Helbling $\mathrm{D}$, et al. Ultrasound assessment of transversus abdominis muscle contraction ratio during abdominal hollowing: a useful tool to distinguish between patients with chronic low back pain and healthy controls? Eur Spine J 2012;21:750-9.

6. Linek P, Saulicz E, Wolny T, Myliwiec A, Kokosz M. Lateral abdominal muscle size at rest and during abdominal drawingin manoeuvre in healthy adolescents. Man Ther 2015;20:117-23.

7. Linek P, Saulicz E, Wolny $\mathrm{T}$, Myśliwiec A. Intra-rater reliability of B-mode ultrasound imaging of the abdominal muscles in healthy adolescents during the active straight leg raise test. PM R 2015;7:53-9.

8. Linek P, Saulicz E, Wolny T, Myśliwiec A. Reliability of B-mode sonography of the abdominal muscles in healthy adolescents in different body positions. J Ultrasound Med 2014;33:1049-56.

9. McMeeken JM, Beith ID, Newham DJ, Milligan P, Critchley DJ. The relationship between EMG and change in thickness of transversus abdominis. Clin Biomech (Bristol, Avon) 2004;19:337-42.

10. Hides J, Wilson S, Stanton W, McMahon S, Keto H, McMahon K, et al. An MRI investigation into the function of the transversus abdominis muscle during drawing-in of the abdominal wall. Spine (Phila Pa 1976) 2006;31:175-8.

11. Maughan RJ, Watson JS, Weir J. Strength and cross-sectional area of human skeletal muscle. J Physiol 1983;338:37-49.

12. Yang HS, Yoo JW, Lee BA, Choi CK, You JH. Inter-tester and intra-tester reliability of ultrasound imaging measurements of abdominal muscles in adolescents with and without idiopathic scoliosis: a case-controlled study. Biomed Mater Eng 2014;24:453-8.

13. Vera-Garcia FJ, Brown SH, Gray JR, McGill SM. Effects of different levels of torso coactivation on trunk muscular and kinematic responses to posteriorly applied sudden loads. Clin Biomech (Bristol, Avon) 2006;21:443-55.

14. Kennelly KP, Stokes MJ. Pattern of asymmetry of paraspinal muscle size in adolescent idiopathic scoliosis examined by real-time ultrasound imaging. A preliminary study. Spine (Phila Pa 1976) 1993;18:913-7.

15. Korovessis P, Koureas G, Zacharatos S, Papazisis Z. Backpacks, back pain, sagittal spinal curves and trunk alignment in adolescents: a logistic and multinomial logistic analysis. Spine (Phila Pa 1976) 2005;30:247-55.

16. Available from: http://www.boneandjoint.org.uk/sites/ default/files/Classification $\% 20$ of $\% 20$ Adolescent $\% 20$ Idiopathic\%20Scoliosis.pdf

17. Negrini S, Aulisa AG, Aulisa L, Circo AB, de Mauroy JC, Durmala J, et al. 2011 SOSORT guidelines: Orthopaedic and Rehabilitation treatment of idiopathic scoliosis during growth. Scoliosis 2012;7:3.

18. Linek P, Saulicz E, Wolny T, Myśliwiec A. Reliability of B-mode ultrasound imaging of the lateral abdominal muscles in healthy children during the active straight leg raise test. Physiotherapy and Health Activity 2014;22:1-7.

19. Linek P, Saulicz E, Wolny T, Myśliwiec A. Assessment of the abdominal muscles at rest and during the abdominal drawing-in manoeuvre in adolescent physically active girls: a case control study. J Sport Health Sci 2017;6:118-24.

20. Linek P. Assessment of the deep abdominal muscles at rest and during the abdominal drawing-in manoeuvre in adolescents practicing volleyball: a case-control study. Isokinet Exerc Sci 2015;23:215-20.

21. Richardson CA, Snijders CJ, Hides JA, Damen L, Pas MS, Storm J. The relation between the transversus abdominis muscles, sacroiliac joint mechanics, and low back pain. Spine (Phila Pa 1976) 2002;27:399-405. 